\title{
OPEN
}

Received: 6 March 2019

Accepted: 4 September 2019

Published online: 27 September 2019

\section{Plasma A $\beta 42$ and Total Tau Predict Cognitive Decline in Amnestic Mild Cognitive Impairment}

\author{
Ting-Bin Chen ${ }^{1,2,3,4}$, Yi-Jung Lee ${ }^{1,5}$, Szu-Ying Lin ${ }^{6}$, Jun-Peng Chen ${ }^{7}$, Chaur-Jong Hu ${ }^{8}$, \\ Pei-Ning Wang ${ }^{9,10,11}$ \& Irene H. Cheng ${ }^{1,11}$
}

Levels of amyloid- $\beta(A \beta)$ and tau peptides in brain have been associated with Alzheimer disease (AD). The current study investigated the abilities of plasma $A \beta 42$ and total-tau (t-tau) levels in predicting cognitive decline in subjects with amnestic mild cognitive impairment (MCI). Plasma A $\beta 42$ and t-tau levels were quantified in 22 participants with amnestic $\mathrm{MCl}$ through immunomagnetic reduction (IMR) assay at baseline. The cognitive performance of participants was measured through neuropsychological tests at baseline and annual follow-up (average follow-up period of 1.5 years). The predictive value of plasma $A \beta 42$ and $t$-tau for cognitive status was evaluated. We found that higher levels of $A \beta 42$ and t-tau are associated with lower episodic verbal memory performance at baseline and cognitive decline over the course of follow-up. While $A \beta 42$ or $t$-tau alone had moderate-to-high discriminatory value in the identification of future cognitive decline, the product of $A \beta 42$ and $t$-tau offered greater differential value. These preliminary results might suggest that high levels of plasma $A \beta 42$ and $t$-tau in amnestic $\mathrm{MCl}$ are associated with later cognitive decline. A further replication with a larger sample over a longer time period to validate and determine their long-term predictive value is warranted.

Mild cognitive impairment (MCI) refers to a transitional state on the continuum of cognitive function between normal aging and mild dementia. Amnestic MCI, characterized by an isolated deficit in episodic memory accompanied by intact general cognitive functioning, has been associated with biomarkers for Alzheimer disease (AD) and is now recognized as a risk factor for $\mathrm{AD}^{1-4}$. Early identification of subsequent cognitive decline in MCI patients is critical for prompt clinical intervention and therapeutic options. Thus, seeking validated biomarkers for risk of cognitive decline is crucial.

To date, validated biomarkers associated with $\mathrm{AD}$ include measurement of brain $\mathrm{A} \beta$ deposition presented by A $\beta 42$ levels in cerebrospinal fluid (CSF), cortical amyloidosis on positron emission tomography (PET), measures of AD-typical neurodegeneration indicated by total-tau ( $\mathrm{t}$-tau) and phosphorylated-tau levels in CSF, hypometabolism on PET, and cerebral atrophy on magnetic resonance imaging (MRI $)^{5}$. Increasing evidence supports an ordered, sequential, non-paralleled temporal trajectory of these five central biomarkers over many years in the continuum of $\mathrm{AD}^{6,7}$. In addition, these biomarkers play significant roles in the definition of $\mathrm{AD}$ pathology $\mathrm{y}^{5,8}$. However, many obstacles, including invasiveness, time, cost, and accessibility to health care services, limit the widespread use of these biomarkers in the clinical setting. Rapid, minimally-invasive, and low-cost blood-based biomarkers are needed to assist in the identification of underlying pathophysiological processes involved in the early stages of $\mathrm{AD}$ and in longitudinal tracking of various disease indicators of progression of $\mathrm{AD}$ pathology.

${ }^{1}$ Institute of Brain Science, National Yang-Ming University, Taipei, Taiwan. ${ }^{2}$ Department of Neurology, Neurological Institute, Taichung Veterans General Hospital, Taichung, Taiwan. ${ }^{3}$ Dementia and Parkinson's Disease Integrated Center, Taichung Veterans General Hospital, Taichung, Taiwan. ${ }^{4}$ Center for Geriatrics and Gerontology, Taichung Veterans General Hospital, Taichung, Taiwan. ${ }^{5}$ Division of Neurology, Department of Medicine, Taipei City Hospital Renai Branch, Taipei, Taiwan. ${ }^{6}$ Taipei Municipal Gan-Dau Hospital, Taipei, Taiwan. ${ }^{7}$ Biostatistics Task Force of Taichung Veterans General Hospital, Taichung, Taiwan. ${ }^{8}$ Department of Neurology, Shuang Ho Hospital, Taipei Medical University, New Taipei City, Taiwan. ${ }^{9}$ Division of General Neurology, Department of Neurological Institute, , Taipei Veterans General Hospital, Taipei, Taiwan. ${ }^{10}$ Aging and Health Research Center, NationalYang-Ming University, Taipei, Taiwan. ${ }^{11}$ Brain Research Center, National Yang-Ming University, Taipei, Taiwan. Correspondence and requests for materials should be addressed to C.-J.H. (email: chaurjongh@tmu.edu.tw) or P.-N.W. (email: linda2860@gmail. com) or I.H.C. (email: hjcheng@ym.edu.tw) 
A $\beta$ accumulation and neurofibrillary tangle formation of phosphorylated-tau precedes full-blown clinical symptoms of $\mathrm{AD}$ by many years to decades, while $\mathrm{A} \beta$ deposition plateaus when patients progress into the clinical $\mathrm{MCI}$ phase of $\mathrm{AD}^{9}$. At this stage of the disease, neurofibrillary tangle formation, increasing gliosis, and progressive neuronal loss are initiated and continue to progress into the clinical state of overt dementia ${ }^{9}$. Accumulating evidence supports that various clearance mechanisms of toxic aggregation of misfolded peptides from the brain to the blood account for a determinate amount of $\mathrm{A} \beta$ and tau in the blood, and therefore, the measurability of low levels of these peptides is only attainable with highly sensitive, robust, and accurate assays ${ }^{10-12}$. Recent researches using ultrasensitive bioanalytic techniques have demonstrated that peripheral and central markers of amyloidosis and neurodegeneration are closely correlated ${ }^{12-15}$. Newly-developing analytic technologies may provide increasing clinical access to simple, convenient, and accurate blood-based biomarkers for high-risk individuals.

Immunomagnetic reduction (IMR) assay is a relatively new high-sensitivity detection technology for the quantification of $A \beta$ and tau. IMR involves antibody-functionalized magnetic nanoparticles in biofluids to measure the reduction in magnetic signals through use of an ultra-high-sensitivity magneto-susceptometer, a superconducting quantum interference device (SQUID) ${ }^{16}$. Previous SQUID-based IMR studies have found that the utilization of a combination of plasma $A \beta 42$ and total tau ( $t$-tau) levels can reliably and accurately identify AD patients in both the prodromal and dementia stage ${ }^{16-18}$. However, the plasma concentrations of $A \beta 42$ and $t$-tau in patients with amnestic MCI have not yet been thoroughly characterized using IMR assays.

The current study used IMR to investigate the predictive value of plasma levels of A $\beta 42$ and t-tau in cognitive decline in subjects with amnestic MCI.

\section{Methods}

Subjects. The current prospective case-control study was conducted from 2015 to 2017. Study participants were consecutively recruited from the memory clinics at Taipei Veterans General Hospital and Shuang Ho Hospital in Taiwan. The inclusion criteria were age of $\geq 60$ years and consent to a longitudinal follow-up period of clinical, neuropsychological, and brain MRI examinations. Prior to testing, written informed consent was obtained from all participants (or their legal guardians) for publication of this research article. The local institutional review board and the ethics committee of Taipei Veterans General Hospital and Shuang Ho Hospital approved the data collection protocol. We confirm that all methods were performed in accordance with the relevant guidelines and regulations by including a statement in the methods section to this effect. All authors have approved the manuscript for submission and gave consent for publication.

Neuropsychological evaluations. All participants were subjected to a standard battery of clinical and comprehensive neuropsychological assessments at baseline and during annual follow-ups. The Mini-Mental Screening Examination (MMSE) ${ }^{19}$ and the Clinical Dementia Rating (CDR) ${ }^{20}$ were used to measure global cognition and severity of dementia, respectively. The Chinese Version Verbal Learning Test (CVVLT) (9 items; total correct trials 1-4, and 10-min delayed free recall $)^{21}$ was administered to evaluate verbal memory performance. Total recall (i.e., the total number of items remembered over 4 trials, CVVLT-T) and free delayed recall (i.e., 10-minute delayed free recall, CVVLT-10) were analysed.

Clinical diagnosis. Clinical diagnosis was based on physical examination, clinical interview, and neuropsychological assessment. A diagnosis of amnestic single-domain MCI was based on the criteria recommended by the National Institute on Aging/Alzheimer's Association (NIA-AA) workgroups in $2011^{22}$. Episodic memory impairment was determined by a score of 1.5 standard deviations below the age- and education-matched normative mean with no accompanying impairments in social or occupational functioning, as assessed by the activities of daily living scale and the instrumental activities of daily living scale. In addition, all MCI subjects had a CDR score of 0.5 .

A formal cognitive test was conducted at annual follow-up visits to ascertain the development of dementia. A diagnosis of probable $\mathrm{AD}$ was made when patients fulfilled the core clinical criteria proposed by the NIA-AA workgroup and had a CDR score of $1^{23}$. Neurological examinations, laboratory tests, and MRI were performed at baseline to exclude non-AD causes of dementia. Exclusion criteria included evidence of other neurological, psychiatric or systemic conditions that may cause cognitive impairment, such as frontotemporal dementia, dementia with Lewy bodies, stroke, vascular dementia, Parkinson's disease, thyroid dysfunction, renal insufficiency, vitamin B12 deficiency, neurosyphilis, alcoholism, and major depression.

The MMSE score was used to measure disease progression. Subjects were dichotomized into a stable group and a declined group according to change in MMSE scores from baseline to follow-up evaluation. Patients with a decrease in MMSE score from baseline were placed in the declined group, while those with no change or an increase in MMSE score were placed in the stable group.

Blood sample processing. Venipuncture was used to collect whole blood into ethylenediaminetetraacetic acid (EDTA)-treated tubes after overnight fasting. Blood was centrifuged (1500-2500 $\times g$, room temperature, $15 \mathrm{~min}$ ) and the supernatant was collected and divided into various aliquots which were maintained at $-80^{\circ} \mathrm{C}$ until the day of testing to avoid multiple freeze/thaw cycles. Frozen plasma samples were delivered on dry ice to MagQu Co., Ltd. (New Taipei City, Taiwan) for IMR assay processing. Assays were performed without knowledge of individual identification or diagnosis.

Genotyping. Apolipoprotein E (ApoE) genotype was determined for all participants by polymerase chain reaction amplification and restriction enzyme digestion following methods described previously ${ }^{24}$.

Immunomagnetic reduction assays. The technical information and the validation accuracy of the IMR assay have been previously well described ${ }^{17,18,25-27}$. The selection of antibodies conjugated to the IMR reagents 


\begin{tabular}{|l|c|c|l|}
\hline & Stable group (N=13) & Declined group (N=9) & $\boldsymbol{p}$ \\
\hline Age, years & $73.0(62.5-80.0)$ & $76.0(69.0-79.5)$ & 0.547 \\
\hline Male & $9(69.2 \%)$ & $4(44.4 \%)$ & 0.384 \\
\hline Education & $12.0(12.0-15.0)$ & $12.0(7.5-16.0)$ & 0.780 \\
\hline ApoE $\varepsilon$ 4 carrier & $3(23.1 \%)$ & $3(33.3 \%)$ & 0.655 \\
\hline MMSE & $27.0(25.0-28.0)$ & $26.0(25.0-26.5)$ & 0.234 \\
\hline CVVLT-T & $27.0(25.8-29.0)$ & $21.0(15.5-22.5)$ & $0.002^{* *}$ \\
\hline CVVLT-10 & $7.0(5.5-8.0)$ & $1.0(0.0-2.0)$ & $0.002^{* *}$ \\
\hline Conversion to AD & $0(0 \%)$ & $4(44.4 \%)$ & $0.017^{*}$ \\
\hline Reversion to normal & $1(7.6 \%)$ & $0(0 \%)$ & 1.000 \\
\hline A $342, \mathrm{pg} / \mathrm{ml}$ & $16.5(15.5-17.1)$ & $18.8(17.3-20.1)$ & $0.021^{*}$ \\
\hline $\mathrm{t}$-tau, $\mathrm{pg} / \mathrm{ml}$ & $18.7(15.6-23.2)$ & $27.2(20.2-39.6)$ & $0.021^{*}$ \\
\hline A $\beta 42 \times \mathrm{t}$-tau, $\mathrm{pg}^{2} / \mathrm{ml}^{2}$ & $325.0(248.1-453.7)$ & $571.3(373.2-778.3)$ & $0.012^{*}$ \\
\hline A $\beta 42 / \mathrm{t}-\mathrm{tau}$ & $0.9(0.7-1.1)$ & $0.6(0.5-1.0)$ & 0.077 \\
\hline
\end{tabular}

Table 1. Participant demographic data $(\mathrm{N}=22)$. Abbreviations: $n$, number of participants; ApoE, apolipoprotein E; MMSE, Mini-Mental State Examination; CVVLT, Chinese Version Verbal Learning Test; $\mathrm{t}$-tau, total tau. Note: The continuous variables, presented as median values and interquartile ranges in parentheses, were calculated by a Mann-Whitney U test. The categorical variables, presented as number of patients and percentage in parentheses, were examined by a Chi-square test. ${ }^{*} p<0.05, * * p<0.01$.

(MagQu Co. Ltd.; catalogue numbers: MF-AB2-0060 and MF-TAU-0060) was based on epitopes, affinity to antigens, ability to conjugate onto MagQu magnetic nanobeads, and ability to provide linearity of standard curves quantified by magnetic signal reduction. For the A $\beta 42$ assay, $60-\mu l$ reagent (MF-AB2-0060, MagQu) was mixed with $60-\mu \mathrm{l}$ plasma at room temperature. For the t-tau assay, $80-\mu \mathrm{l}$ reagent (MF-TAU-0060, MagQu) was mixed with 40- $\mu$ l plasma. A SQUID-based alternative current magnetosusceptometer (model XacPro-S, MagQu Co., New Taipei City, Taiwan) was used for analysis. The magnetosusceptometer detects magnetic signal changes during the course of antigen and antibody interactions, expressed as percentage reduction of immunomagnetic signals (IMR \%), which are then converted to sample concentrations using values from the standard curves of the respective analytes. The reduction of oscillation detected by SQUID corresponds to the amount of analytes bound to the antibodies. Duplicates were conducted for each biomarker assay.

Statistical analysis. All statistical analyses were performed in SPSS version 22.0 for Windows (SPSS Inc., Chicago, IL). A $p$-value $<0.05$ was considered significant. All variables were analysed through non-parametric methods. For continuous variables, differences between the stable group and the declined group were detected with a Mann-Whitney U test. For categorical variables, the Chi-square test was used. Spearman's rank correlation coefficient was used to explore the correlation between plasma biomarker levels and cognitive performance (i.e., MMSE and CVVLT). Receiver operating characteristic (ROC) analyses were computed to identify possible useful cut-off points of single A $\beta 42$ or t-tau analytes, or their combinations (ratio or product) to further characterize discriminatory properties between the stable and declined groups. Additional analysis with Cox proportional regression (enter method) was carried out to investigate the power of biomarker levels and respective cut-off values in the prediction of cognitive decline in MCI, in terms of hazard ratios, with and without adjusting by age, gender, years of education, and ApoE $\varepsilon 4$ carrier status.

\section{Results}

Baseline demographic data. At baseline, 22 subjects with amnestic MCI were enrolled in the study. The average follow-up period was 18.6 months (range, 9.7-25.8 months; median, 21.3 months). The demographic characteristics of the subjects are presented in Table 1. The annual conversion rate from MCI to AD was $14.7 \%$. There were no significant differences in age, gender, years of education, proportions of ApoE $\varepsilon 4$ carrier, or baseline MMSE scores between the stable and declined groups. The declined group had poorer baseline performance on the CVVLT, higher levels of plasma biomarkers, and greater incidence of conversion to AD during follow-up. In addition, the MMSE decreased by an average of 2.2 points per year in the declined group, while the stable group showed minimal change (0.84 point/year).

Association of plasma biomarkers with MMSE and CVVLT at baseline. There was a significant correlation between the cognitive performance on verbal memory test and plasma biomarker levels (Table 2). The CVVLT-T scores were significantly correlated with A $\beta 42$ levels $(\mathrm{r}=-0.555, \mathrm{p}=0.032), \mathrm{t}$ - $\mathrm{tau}(\mathrm{r}=-0.519$, $\mathrm{p}=0.047)$, and the product of $\mathrm{A} \beta 42$ and $\mathrm{t}$-tau levels $(\mathrm{A} \beta 42 \times \mathrm{t}-\mathrm{tau}, \mathrm{r}=-0.571, \mathrm{p}=0.026)$, while the CVVLT-10 scores were significantly correlated with $\mathrm{A} \beta 42 \times \mathrm{t}$-tau $(\mathrm{r}=-0.516, \mathrm{p}=0.049)$ (Table 2 and Fig. 1).

Association of plasma biomarkers with annual changes in MMSE and CVVLT. There was a significant negative correlation between the annual change in MMSE scores and plasma biomarker levels (Table 2). The annual change in MMSE scores was correlated with the A $\beta 42$ levels $(r=-0.512, p=0.015)$ and $A \beta 42 \times t$-tau $(\mathrm{r}=-0.429, \mathrm{p}=0.046)$ (Table 2 and Fig. 2). However, there was no significant correlation between plasma biomarkers and annual changes in CVVLT-T and CVVLT-10 scores. 


\begin{tabular}{|c|c|c|c|c|c|c|}
\hline \multirow{2}{*}{\begin{tabular}{|l|} 
Baseline \\
Biomarkers
\end{tabular}} & \multicolumn{2}{|l|}{ MMSE } & \multicolumn{2}{|c|}{ CVVLT-T } & \multicolumn{2}{|c|}{ CVVLT-10 } \\
\hline & $\mathbf{r}_{\mathrm{s}}$ & $p$ & $\mathbf{r}_{\mathrm{s}}$ & $p$ & $\mathbf{r}_{\mathrm{s}}$ & $p$ \\
\hline $\mathrm{A} \beta 42$ & -0.024 & 0.917 & -0.555 & $0.032 *$ & -0.393 & 0.147 \\
\hline $\mathrm{t}$-tau & -0.138 & 0.539 & -0.519 & $0.047^{*}$ & -0.512 & 0.051 \\
\hline A $342 \times$ t-tau & -0.061 & 0.786 & -0.571 & $0.026^{*}$ & -0.516 & $0.049^{*}$ \\
\hline $\mathrm{A} \beta 342 / \mathrm{t}-\mathrm{tau}$ & 0.160 & 0.478 & 0.499 & 0.058 & 0.491 & 0.063 \\
\hline Annual changes & \multicolumn{2}{|l|}{ MMSE } & \multicolumn{2}{|c|}{ CVVLT-T } & \multicolumn{2}{|c|}{ CVVLT-10 } \\
\hline Biomarkers & $\mathbf{r}_{\mathrm{s}}$ & $p$ & $\mathbf{r}_{\mathrm{s}}$ & $p$ & $\mathbf{r}_{\mathrm{s}}$ & $p$ \\
\hline $\mathrm{A} \beta 42$ & -0.512 & 0.015* & 0.009 & 0.975 & -0.022 & 0.939 \\
\hline t-tau & -0.376 & 0.085 & -0.070 & 0.805 & 0.005 & 0.985 \\
\hline A $\beta 42 \times$ t-tau & -0.429 & 0.046* & -0.077 & 0.785 & -0.029 & 0.919 \\
\hline $\mathrm{A} \beta 42 / \mathrm{t}$-tau & 0.244 & 0.273 & 0.100 & 0.723 & 0.040 & 0.888 \\
\hline
\end{tabular}

Table 2. Association between baseline plasma biomarkers and MMSE and CVVLT scores at baseline and follow-up ( $\mathrm{N}=22)$. Abbreviations: MMSE, Mini-Mental State Examination; CVVLT, Chinese Version Verbal Learning Test; t-tau, total tau. Note: Spearman's rank correlation coefficient was used to explore the correlation between plasma biomarker levels and MMSE and CVVLT scores at baseline and follow-up. ${ }^{*} p<0.05$.

(a)

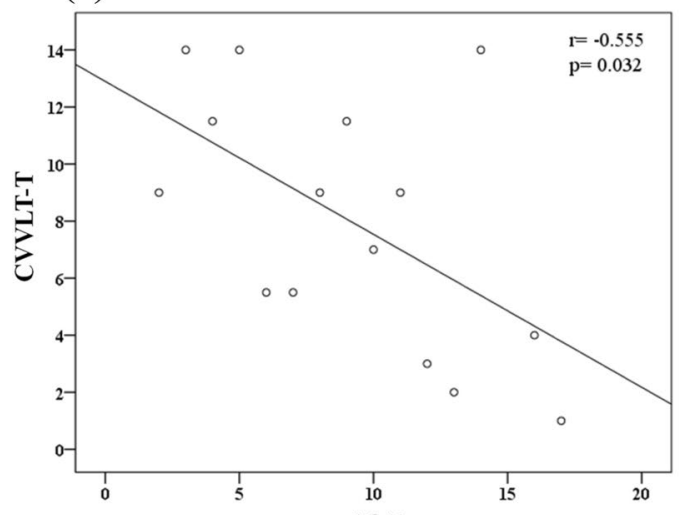

(c)

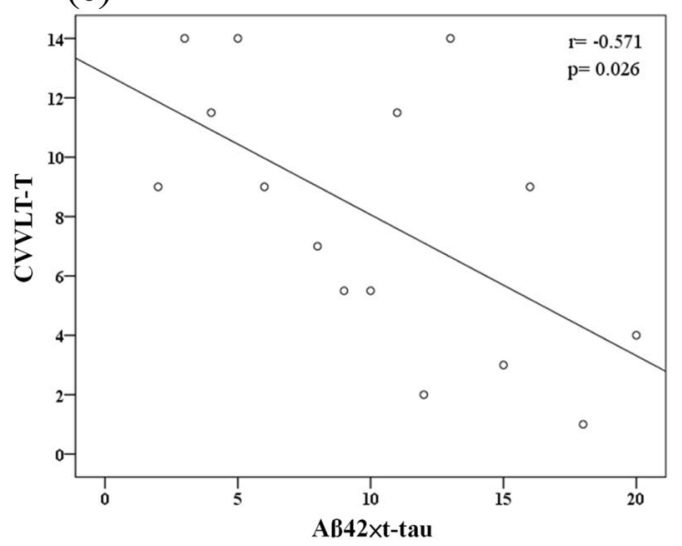

(b)

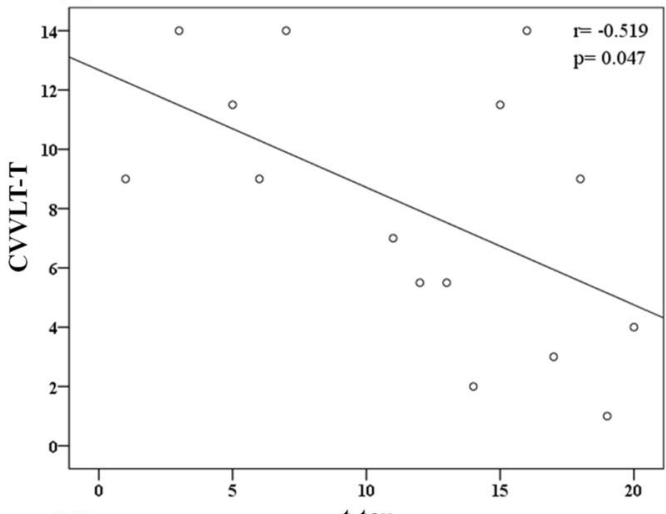

(d)

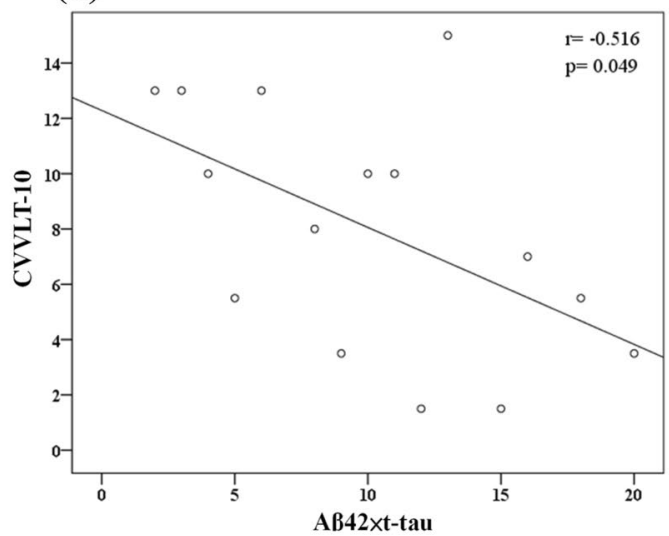

Figure 1. Scatterplots of the associations between baseline CVVLT scores and plasma biomarker levels (pg/ml).

ROC analysis for prediction of cognitive decline in $\mathrm{MCl}$. The discriminatory properties of plasma biomarkers for prediction of cognitive decline were investigated using ROC analyses of the stable group versus the declined group in order to obtain cut-off values at the greatest area under the ROC curves (AUC). Optimal cut-off values of $16.8 \mathrm{pg} / \mathrm{ml}$ for A $\beta 42,25.4 \mathrm{pg} / \mathrm{ml}$ for $\mathrm{t}$-tau, and $465.1 \mathrm{pg}^{2} / \mathrm{ml}^{2}$ for A $\beta 42 \times \mathrm{t}$-tau were identified in the differentiation of stable and declined participants. A $\beta 42 \times \mathrm{t}$-tau obtained the highest AUC $(0.82, \mathrm{p}<0.001)$. The sensitivity (SN), specificity (SP), and AUC of single A $\beta 42$ and t-tau were enhanced by the computed product of $A \beta 42$ and t-tau. Detailed results for AUC, cut-off values, SN, SP, accuracy (AC), and positive likelihood ratio $(\mathrm{LR}+)$ are displayed in Table 3 and Fig. 3.

Association of plasma biomarkers with cognitive decline in $\mathrm{MCl}$. The relationship between plasma biomarkers and incidence of cognitive decline during the follow-up visits was examined through Cox regression 
(a)

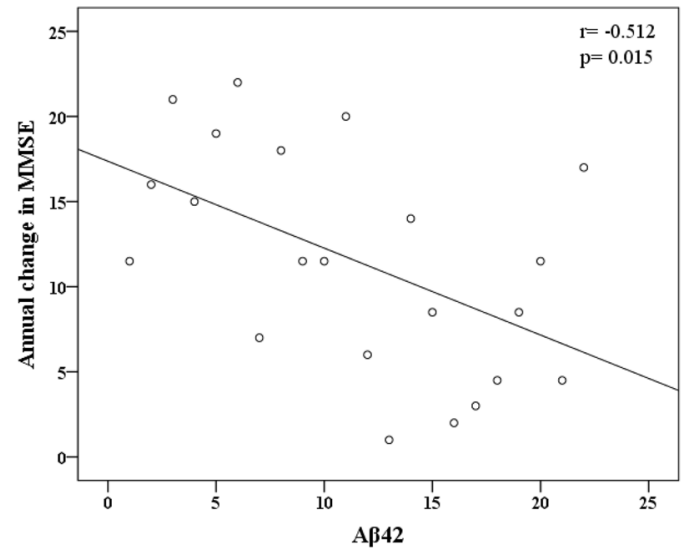

(b)

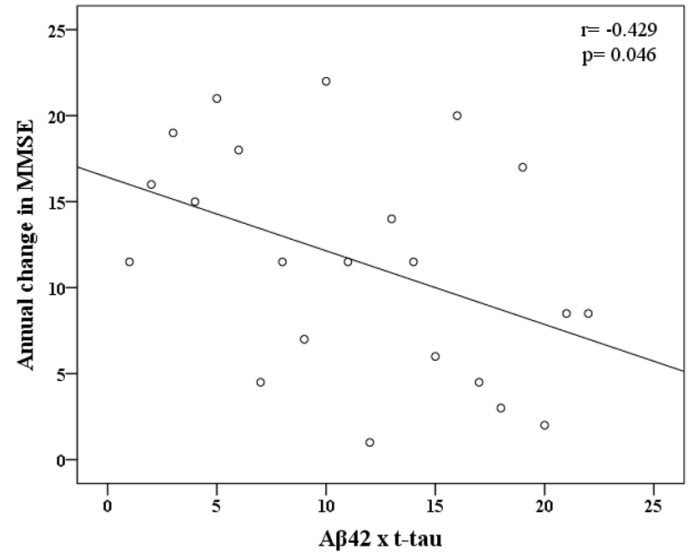

Figure 2. Scatterplots of the associations between annual change in MMSE scores and plasma biomarker levels $(\mathrm{pg} / \mathrm{ml})$.

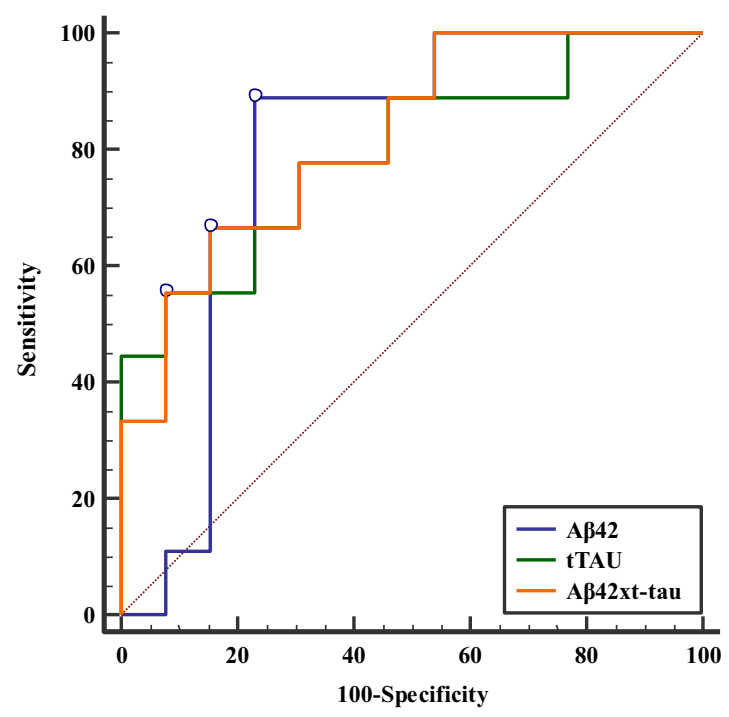

Figure 3. ROC curve for discriminating the stable group from the decline group using A $\beta 42$ level, t-tau level, and $\mathrm{A} \beta 42 \times \mathrm{t}$-tau value as diagnostic parameters.

\begin{tabular}{|l|l|c|c|l|l|l|l|}
\hline Variables & AUC $(\mathbf{9 5} \% \mathbf{C I})$ & \multicolumn{1}{l}{$\boldsymbol{p}$} & Cutoff & SN (\%) & SP (\%) & AC (\%) & LR+ \\
\hline A 342 & $0.80(0.57-0.94)$ & $0.005^{* *}$ & $>16.8$ & 88.89 & 76.92 & 81.82 & 3.85 \\
\hline t-tau & $0.80(0.57-0.94)$ & $0.004 * *$ & $>25.4$ & 55.56 & 92.31 & 77.27 & 7.22 \\
\hline A $342 \times$ t-tau & $0.82(0.60-0.95)$ & $<0.001 * *$ & $>465.1$ & 66.67 & 84.62 & 77.27 & 4.33 \\
\hline A $342 /$ t-tau & $0.72(0.49-0.88)$ & 0.087 & $\leq 0.63$ & 55.56 & 92.31 & 77.27 & 7.22 \\
\hline
\end{tabular}

Table 3. ROC analysis for cognitive decline in MCI. Abbreviations: AUC, area under the receiver operating characteristic curve; CI, confidence interval; SN, sensitivity; SP, specificity; AC, accuracy; LR+, positive likelihood ratio. $* * p<0.01$.

analyses using the optimal cut-offs as described above. The results of the unadjusted and adjusted Cox regression models for estimating the relative risk of developing cognitive decline in MCI patients are listed in Table 4. All markers, except A $\beta 42 / t$-tau, were significantly associated with cognitive deterioration in MCI patients. These findings were subsequently confirmed in the adjusted model. All adjusted hazard ratios (aHRs) increased after controlling for age, gender, years of education, and ApoE $\varepsilon 4$ carrier status. Participants with A $\beta 42>16.8 \mathrm{pg} / \mathrm{ml}$ (aHR, 16.84; 95\% CI, 1.88-150.45; $\mathrm{p}=0.011), \mathrm{A} \beta 42 \times \mathrm{t}$-tau values $>465.1 \mathrm{pg}^{2} / \mathrm{ml}^{2}(\mathrm{aHR}, 7.14$; 95\% CI, $1.57-$ $32.55 ; \mathrm{p}=0.011)$, and t-tau levels $>25.4 \mathrm{pg} / \mathrm{ml}(\mathrm{aHR}, 5.19 ; 95 \% \mathrm{CI}, 1.20-22.53 ; \mathrm{p}=0.028)$ were at increased risk for future cognitive decline. 


\begin{tabular}{|c|c|c|c|c|c|c|}
\hline & \multicolumn{3}{|c|}{ Unadjusted } & \multicolumn{3}{|c|}{ Adjusted* } \\
\hline & HR & 95\% CI & $p$ & HR & 95\% CI & $p$ \\
\hline \multicolumn{7}{|c|}{$\mathrm{A} \beta 42, \mathrm{pg} / \mathrm{ml}$} \\
\hline$\leq 16.8$ & 1.00 & & & 1.00 & & \\
\hline$>16.8$ & 11.71 & $(1.45-94.64)$ & $0.021^{*}$ & 16.84 & $(1.88-150.45)$ & $0.011 *$ \\
\hline \multicolumn{7}{|c|}{ t-tau, pg/ml } \\
\hline$\leq 25.4$ & 1.00 & & & 1.00 & & \\
\hline$>25.4$ & 4.51 & $(1.19-17.13)$ & $0.027^{*}$ & 5.19 & $(1.20-22.53)$ & $0.028 *$ \\
\hline \multicolumn{7}{|c|}{$\mathrm{A} \beta 42 \times \mathrm{t}-\mathrm{tau}, \mathrm{pg}^{2} / \mathrm{ml}^{2}$} \\
\hline$\leq 465.1$ & 1.00 & & & 1.00 & & \\
\hline$>465.1$ & 6.08 & $(1.45-25.41)$ & $0.013^{*}$ & 7.14 & $(1.57-32.55)$ & $0.011 *$ \\
\hline \multicolumn{7}{|c|}{ A $\beta 42 /$ t-tau } \\
\hline$\leq 0.63$ & 1.00 & & & 1.00 & & \\
\hline$>0.63$ & 0.28 & $(0.08-1.07)$ & 0.063 & 0.29 & $(0.07-1.17)$ & 0.083 \\
\hline
\end{tabular}

Table 4. Cox regression analysis of predictors for cognitive decline in MCI. Abbreviations: HR, hazard ratio; $\mathrm{CI}$, confidence interval. *Models were adjusted for age, gender, years of education, and APOE $\varepsilon 4$ carrier status. $* p<0.05$.

\section{Discussion}

The current preliminary study assessed the predictive power of plasma A $\beta 42$ and $t$-tau in cognitive decline in patients with amnestic MCI. IMR technology, a high-sensitivity assay platform, was used to reliably detect ultra-low concentrations of $\mathrm{A} \beta 42$ and $\mathrm{t}$-tau in blood. Higher levels of plasma biomarkers (i.e., A $\beta 42$, $t$-tau and $\mathrm{A} \beta 42 \times \mathrm{t}$-tau) were found in participants who showed cognitive decline (the declined group) compared to those who did not (the stable group) and were associated with lower episodic verbal memory performance at baseline and a greater annual decrease in MMSE score. A $\beta 42$ and t-tau had moderate-to-high discriminatory ability (AUC > 0.70), while combining the two biomarkers (i.e., A $\beta 42 \times \mathrm{t}$-tau) added further differential value. After adjusting for relevant demographic covariates (age, gender, years of education, and ApoE $\varepsilon 4$ carrier status), $\mathrm{A} \beta 42, \mathrm{t}$-tau, and $\mathrm{A} \beta 42 \times \mathrm{t}$-tau proved to be strong predictive biomarkers for future cognitive decline in MCI. Collectively, these findings suggest that both $\mathrm{A} \beta 42$ and $\mathrm{t}$-tau are potential predictors for monitoring progressive cognitive decline in the MCI stage of AD.

During $\mathrm{AD}$ progression, $\mathrm{A} \beta$ and tau proteins are readily excreted from the brain into the peripheral blood through disruption of blood-brain barrier and receptor-mediated mechanisms ${ }^{10,12,28}$. A $\beta$ and tau are therefore detectable in plasma through highly sensitive and accurate analytic techniques. Until now, there has been great progress in the development of novel techniques to measure $\mathrm{A} \beta$ and tau in the blood (reviewed by others ${ }^{29-32}$ ). Recent studies using ultrasensitive analytical assays, such as IMR, single-molecule array (SIMOA), and immunoprecipitation mass spectrometry (IP-MS) approaches, have obtained enhanced technical accuracy of biomarkers in blood samples. IMR assays have the capacity to quantify plasma $\mathrm{A} \beta$ and tau at levels as low as $1-10 \mathrm{pg} / \mathrm{ml}^{25,33,34}$. Plasma A $\beta 42$ levels lower than the detection limit of conventional A $\beta 42$ enzyme-linked immunosorbent assays (ELISAs) are likely to be encountered in the MCI stage and bias parameter estimates at or below the detection threshold of conventional ELISAs (e.g., false negatives). These assays detect an increase in A $\beta 42$ signal in prodromal AD with high accuracy (nearly 85\%), and using an $\mathrm{A} \beta 42 \times \mathrm{t}$-tau of $382.68 \mathrm{pg}^{2} / \mathrm{ml}^{2}$ as the cut-off value was $92 \%$ accurate in the identification of $\mathrm{AD}^{16,18}$. SIMOA assays are capable of detecting $\mathrm{A} \beta 42$ at subpicogram/ $\mathrm{ml}$ levels (near $0.04 \mathrm{pg} / \mathrm{ml}$ ), and have shown that $\mathrm{A} \beta 42$ levels and $\mathrm{A} \beta 42 / \mathrm{A} \beta 40$ ratios are correlated with CSF levels and $\mathrm{A} \beta$ burden on PET and that $\mathrm{A} \beta 42 / \mathrm{A} \beta 40$ ratios are associated with risk of MCI or AD in cognitively normal subjects $^{13,35}$. Recent IP-MS study has demonstrated that alterations in plasma and CSF A $\beta 42$ levels show similar kinetics and that plasma $\mathrm{A} \beta 42$ levels correlate with both CSF A $\beta 42$ levels and $\mathrm{A} \beta$ burden as revealed by $\mathrm{A} \beta \mathrm{PET}^{12}$. Conversely, another IP-MS study reported that a decrease in $A \beta 42$ levels and $A \beta 42 / A \beta 40$ ratios predicted $A \beta$ PET positivity in AD, MCI, and cognitively normal populations with a high accuracy (nearly $90 \%)^{14}$. Moreover, a newly-developed antibody-based infrared sensor method has shown that the secondary structure change of plasma $\mathrm{A} \beta$ peptides correlates with CSF AD biomarkers and A $\beta$ PET imaging ${ }^{36}$. The contradictory findings across the above studies may be partially explained by the various detection capacities of $A \beta$ aggregates or $A \beta$ bound to other proteins using different bioanalytical platforms.

Prior studies have shown t-tau levels to be significantly higher in AD than healthy controls, but there is considerable overlap across the diagnostic groups using both $\mathrm{IMR}^{18}$ and SIMOA ${ }^{37}$ methods. A previous IMR study found a negative association between plasma t-tau and volume of total gray matter, hippocampal volume, amygdale volume, and cognitive measures of logical memory, visual reproduction, and verbal fluency in MCI or early $\mathrm{AD}^{26}$. Recent studies using SIMOA have emphasized the role of plasma t-tau/A $\beta 42$ in the prediction of brain tau pathology and neurodegeneration in $\mathrm{AD}$ and have also demonstrated a positive correlation of phospho-tau181 levels with clinical $\mathrm{AD}$ severity and deposition of tau and $\mathrm{A} \beta$ detected by $\mathrm{PET}^{15,38}$.

Unlike the findings from studies using conventional assay technologies, studies using ultrahigh-sensitivity assay technologies have consistently shown significant correlations between peripheral and central markers of amyloidosis and neurodegeneration indicative of brain AD pathology. Due to the differing findings reported 
above, further studies using larger longitudinal cohorts of participants are necessary to determine the practicability of plasma biomarkers in the clinical settings.

Studies that have explored plasma A 342 in the pre-dementia stage of AD have yielded inconsistent results and a broad spectrum of changes. Prior studies have shown that plasma A $\beta 42$ levels are higher in non-demented participants with a greater risk for dementia and $\mathrm{AD}^{39,40}$ and in subjects with $\mathrm{MCI}$, compared to healthy controls and $\mathrm{AD}$ patients ${ }^{41-43}$. Other studies have shown that plasma $\mathrm{A} \beta 42$ concentrations increased prior to dementia onset in familial $\mathrm{AD}$ with presenilin or amyloid precursor protein (APP) mutations ${ }^{44,45}$, in Down syndrome with APP triplication $^{46,47}$, and in first-degree relatives of AD patients, who are at an increased risk of developing the disease ${ }^{48-50}$. Conflicting studies have found decreased plasma $A \beta 42$ or $A \beta 42 / A \beta 40$ ratio associated with the progression from healthy controls and $\mathrm{MCI}$ to $\mathrm{AD}$ and cognitive decline in a population at risk for $\mathrm{AD}^{51-55}$. Moreover, one study found no difference in plasma A $\beta 42$ levels between healthy controls, subjects with stable MCI, MCI progressors, and $\mathrm{AD}$ patients ${ }^{56}$.

Studies on t-tau plasma levels have also yielded inconsistent results. For example, prior studies have shown that compared with cognitively normal controls, $t$-tau levels increase in $\mathrm{AD}$, but not in $\mathrm{MCI}^{37,57}$, rise in $\mathrm{MCI}^{58}$, elevate in both $\mathrm{MCI}$ and early $\mathrm{AD}^{26}$, do not change in both $\mathrm{MCI}$ and $\mathrm{AD}^{59}$, and decrease in $\mathrm{MCI}$ and $\mathrm{AD}^{60}$. The discrepancies between findings for these biomarkers may be caused by varied quantification methods (e.g., digital array technology, SIMOA, IMR assay, and ELISA), platform-related factors (e.g., matrix effect, interference, and epitope masking), confounding clinical and demographic factors (e.g., age, renal/hepatic function, comorbidities, dietary status, study cohort, disease stage, and follow-up duration), and the absence of verification for brain $A \beta$ or tau accumulation. Despite the heterogeneity of results, plasma markers of AD pathology remain a focus of interest in predicting development of cognitive decline in at-risk subjects because they are simple, inexpensive, and non-invasive, all of which are significant merits for population-based screening tools.

The present study identified significant negative associations between levels of plasma $A \beta 42, t-t a u$, and A $342 \times \mathrm{t}$-tau and cognitive measures of episodic verbal memory and annual change in MMSE scores. These findings are in line with previous studies showing an inverse relationship between levels of $A \beta 42$ and $t$-tau and cognitive performance in the pre-dementia stage $\mathrm{e}^{26,43,57,61-64}$. In addition, at follow-up (with a mean period of 1.5 years), we found that $\mathrm{A} \beta 42$ levels increase in blood during the clinical phase of amnesic MCI. This finding is extremely valuable from a clinical perspective, as the ability to identify at-risk individuals 1-2 years prior to a clinical diagnosis of $\mathrm{AD}$ can significantly improve the quality of care and allow patients and their families to plan and to receive timely practical information and support, and to initiate suitable clinical intervention.

Both plasma $\mathrm{A} \beta 42$ and t-tau can serve as a window, providing insights into brain functioning involved in verbal memory and global cognition during the natural progression of amnestic MCI. The findings from the present study along with results from prior studies reviewed above, showing associations of plasma biomarkers with CSF biomarkers, neuroimaging abnormalities and cognitive measures, indicate that increased plasma A $\beta 42$ and t-tau are likely to occur during the progressive course of MCI in parallel with cognitive decline before the clinical onset of $\mathrm{AD}$. These biomarkers, therefore, reflect the pathophysiological process of $\mathrm{AD}$ that commences years before overt cognitive functions worsen.

ROC curves were used to determine the cut-off values for each plasma biomarker and their combination to differentiate participants in the decline group from the stable group. This analysis indicated that A $\beta 42$ alone exhibited high SN, SP, and AC in the identification of MCI patients with cognitive decline. Higher plasma A $\beta 42$ $(>16.8 \mathrm{pg} / \mathrm{ml})$ and $\mathrm{t}$-tau $(>25.4 \mathrm{pg} / \mathrm{ml})$ at the onset of the study were associated with a $5-17$-fold increased risk of cognitive decline. Since the concentrations of both $A \beta 42$ and t-tau protein are higher in the decline group than in the stable group, it is reasonable to use the composite marker of $\mathrm{A} \beta 42 \times \mathrm{t}$-tau as a potential prognostic parameter to improve the discriminatory power. Single A 342 and t-tau had moderate-to-high predictive ability $(A U C>0.70)$, while the combination of the two biomarkers (i.e., A $\beta 42 \times$ t-tau) showed an incremental benefit to between-group differentiation. A $\beta 42 \times \mathrm{t}$-tau achieved a higher AUC (0.82) and acceptable SN (66.67\%) and SP (84.62\%). Although this combination did not increase LR+, it retained adequate predictive performance and revealed a sufficient discrimination power between the two groups $(\mathrm{LR}+=4.33)$. The cut-off values for levels of A 342 and $t$-tau used in the current study to differentiate groups are in agreement with other studies performed with non-demented elderly subjects ${ }^{16-18}$.

After controlling for the demographic covariates, $\mathrm{A} \beta 42$, $\mathrm{t}$-tau, and $\mathrm{A} \beta 42 \times \mathrm{t}$-tau showed strong promise as predictive biomarkers for future cognitive decline in the MCI stage, whereas A $\beta 42 / t$-tau ratio did not have predictive value. Using SIMOA and xMAP technology, A $\beta 42$, which positively correlated with CSF A $\beta 42$ but negatively with CSF t-tau, was higher in the MCI stage than in the AD stage, while t-tau was positively associated with the burden of brain tau deposition on tau PET across the AD spectrum, that is, A $\beta 42$ elevates during the MCI stage and reaches a plateau before the demented stage, while t-tau increases with $\mathrm{AD}$-associated tau pathology $\mathrm{y}^{15,43}$. Therefore, the product of $\mathrm{A} \beta 42$ and $\mathrm{t}$-tau improved the clinical usefulness in the MCI stage, which was supported by AUC analyses of predicting accuracy for developing cognitive decline. We consider that utilization of plasma $\mathrm{A} \beta 42$ and $\mathrm{t}$-tau together is superior to absolute levels of individual peptides and to the A $\beta 42 / \mathrm{t}$-tau ratio as markers of clinical deterioration in MCI. As this study evaluated a short time period of about 1.5 years in a small cohort of 22 subjects with MCI, our preliminary data might only infer a possible association of plasma A $\beta 42$ and t-tau with an imminent risk of conversion to AD. This result warrants a further replication with a larger sample over a longer time period to validate and determine their long-term predictive value in detecting AD. Further longitudinal studies are needed to assess whether high plasma $\mathrm{A} \beta 42$ and $\mathrm{t}$-tau are clinically useful prognostic biomarkers for $\mathrm{AD}$. Whether the plasma biomarkers alone, in combination, or with other biomarkers, improve diagnostic efficiency, patient discrimination, and disease monitoring along the normal to $\mathrm{AD}$ continuum deserves additional researches.

While the current study contributes valuable findings, there are some limitations that should be considered. First, the sample size was relatively small. A larger, more diverse population with a longer follow-up period is 
needed to support the generalization of these results and to establish the significance of plasma biomarkers in the identification of individuals at risk for cognitive deterioration in the MCI stage of AD. Second, a larger sample size is required to validate the measures of sensitivity, specificity, and accuracy in the ROC analysis as well as obtain the optimal cut-off values of $A \beta 42$ and t-tau. Third, MCI duration was not controlled for in the present study and may have affected plasma biomarker levels. Forth, the current study did not perform A $\beta$ PET or provide neuropathological confirmation of dementia. Finally, comorbidities that may affect plasma biomarker levels were not covaried in the present study.

In conclusion, consistent with previous studies showing promising discriminatory power of plasma A $\beta 42$ and $\mathrm{t}$-tau assays with IMR technology over the normal-MCI-AD cognitive spectrum ${ }^{16-18}$, the overall findings of the current preliminary study might support that higher plasma $\mathrm{A} \beta 42$ and t-tau levels in the MCI stage are predictors of greater risk for the development of cognitive decline in the pre-dementia stage of AD. A $\beta 42$ and t-tau might therefore be considered as markers reflecting disease severity in monitoring patients with early memory disorders. Combining the two plasma biomarkers with other markers might help to identify MCI subjects at risk for $\mathrm{AD}$. The early detection of patients at risk will be beneficial for early and preventive pharmacological intervention.

\section{References}

1. McGuinness, B., Barrett, S. L., McIlvenna, J., Passmore, A. P. \& Shorter, G. W. Predicting conversion to dementia in a memory clinic: A standard clinical approach compared with an empirically defined clustering method (latent profile analysis) for mild cognitive impairment subtyping. Alzheimer's \& dementia (Amsterdam, Netherlands) 1, 447-454, https://doi.org/10.1016/j.dadm.2015.10.003 (2015).

2. Petersen, R. C. Mild Cognitive Impairment. Continuum (Minneapolis, Minn.) 22, 404-418, https://doi.org/10.1212/ con.0000000000000313 (2016).

3. Hughes, T. F., Snitz, B. E. \& Ganguli, M. Should mild cognitive impairment be subtyped? Current opinion in psychiatry 24, 237-242, https://doi.org/10.1097/YCO.0b013e328344696b (2011).

4. Brambati, S. M. et al. Single- and multiple-domain amnestic mild cognitive impairment: two sides of the same coin? Dementia and geriatric cognitive disorders 28, 541-549, https://doi.org/10.1159/000255240 (2009).

5. Jack, C. R. Jr. et al. NIA-AA Research Framework: Toward a biological definition of Alzheimer's disease. Alzheimer's \& dementia: the journal of the Alzheimer's Association 14, 535-562, https://doi.org/10.1016/j.jalz.2018.02.018 (2018).

6. Jack, C. R. Jr. \& Holtzman, D. M. Biomarker modeling of Alzheimer's disease. Neuron 80, 1347-1358, https://doi.org/10.1016/j. neuron.2013.12.003 (2013)

7. Jack, C. R. Jr. et al. Tracking pathophysiological processes in Alzheimer's disease: an updated hypothetical model of dynamic biomarkers. The Lancet. Neurology 12, 207-216, https://doi.org/10.1016/s1474-4422(12)70291-0 (2013).

8. Dubois, B. et al. Advancing research diagnostic criteria for Alzheimer's disease: the IWG-2 criteria. The Lancet. Neurology 13, 614-629, https://doi.org/10.1016/s1474-4422(14)70090-0 (2014).

9. Jack, C. R. Jr. et al. Hypothetical model of dynamic biomarkers of the Alzheimer's pathological cascade. The Lancet. Neurology 9 , 119-128, https://doi.org/10.1016/s1474-4422(09)70299-6 (2010).

10. Tarasoff-Conway, J. M. et al. Clearance systems in the brain-implications for Alzheimer disease. Nature reviews. Neurology 11, 457-470, https://doi.org/10.1038/nrneurol.2015.119(2015).

11. Yanamandra, K. et al. Anti-tau antibody administration increases plasma tau in transgenic mice and patients with tauopathy. Science translational medicine 9, https://doi.org/10.1126/scitranslmed.aal2029 (2017).

12. Ovod, V. et al. Amyloid beta concentrations and stable isotope labeling kinetics of human plasma specific to central nervous system amyloidosis. Alzheimer's \& dementia: the journal of the Alzheimer's Association 13, 841-849, https://doi.org/10.1016/j. jalz.2017.06.2266 (2017).

13. Janelidze, S. et al. Plasma beta-amyloid in Alzheimer's disease and vascular disease. Scientific reports 6, 26801, https://doi. org/10.1038/srep26801 (2016).

14. Nakamura, A. et al. High performance plasma amyloid- $\beta$ biomarkers for Alzheimer's disease. Nature 554, 249, https://doi. org/10.1038/nature25456 (2018).

15. Park, J. C. et al. Plasma tau/amyloid-beta1-42 ratio predicts brain tau deposition and neurodegeneration in Alzheimer's disease. Brain: a journal of neurology, https://doi.org/10.1093/brain/awy347 (2019).

16. Yang, S. Y., Chiu, M. J., Chen, T. F. \& Horng, H. E. Detection of Plasma Biomarkers Using Immunomagnetic Reduction: A Promising Method for the Early Diagnosis of Alzheimer's Disease. Neurology and therapy 6, 37-56, https://doi.org/10.1007/s40120-017-0075-7 (2017).

17. Chiu, M. J. et al. Combined plasma biomarkers for diagnosing mild cognition impairment and Alzheimer's disease. ACS chemical neuroscience 4, 1530-1536, https://doi.org/10.1021/cn400129p (2013).

18. Lue, L. F. et al. Plasma Levels of Abeta42 and Tau Identified Probable Alzheimer's Dementia: Findings in Two Cohorts. Frontiers in aging neuroscience 9,226 , https://doi.org/10.3389/fnagi.2017.00226 (2017).

19. Folstein, M. F., Folstein, S. E. \& McHugh, P. R. Mini-mental state. A practical method for grading the cognitive state of patients for the clinician. Journal of psychiatric research 12, 189-198 (1975).

20. Hughes, C. P., Berg, L., Danziger, W. L., Coben, L. A. \& Martin, R. L. A new clinical scale for the staging of dementia. The British journal of psychiatry: the journal of mental science 140, 566-572 (1982).

21. Chang, C. C. et al. Validating the Chinese version of the Verbal Learning Test for screening Alzheimer's disease. Journal of the International Neuropsychological Society: JINS 16, 244-251, https://doi.org/10.1017/s1355617709991184 (2010).

22. Albert, M. S. et al. The diagnosis of mild cognitive impairment due to Alzheimer's disease: recommendations from the National Institute on Aging-Alzheimer's Association workgroups on diagnostic guidelines for Alzheimer's disease. Alzheimer's \& dementia: the journal of the Alzheimer's Association 7, 270-279, https://doi.org/10.1016/j.jalz.2011.03.008 (2011).

23. McKhann, G. M. et al. The diagnosis of dementia due to Alzheimer's disease: recommendations from the National Institute on Aging-Alzheimer's Association workgroups on diagnostic guidelines for Alzheimer's disease. Alzheimer's \& dementia: the journal of the Alzheimer's Association 7, 263-269, https://doi.org/10.1016/j.jalz.2011.03.005 (2011).

24. Liu, H. C. et al. ApoE genotype in relation to AD and cholesterol: a study of 2,326 Chinese adults. Neurology 53, $962-966$ (1999).

25. Yang, C. C. et al. Biofunctionalized magnetic nanoparticles for specifically detecting biomarkers of Alzheimer's disease in vitro. ACS chemical neuroscience 2, 500-505, https://doi.org/10.1021/cn200028j (2011).

26. Chiu, M. J. et al. Plasma tau as a window to the brain-negative associations with brain volume and memory function in mild cognitive impairment and early Alzheimer's disease. Human brain mapping 35, 3132-3142, https://doi.org/10.1002/hbm.22390 (2014).

27. Tzen, K. Y. et al. Plasma Abeta but not tau is related to brain PiB retention in early Alzheimer's disease. ACS chemical neuroscience 5, 830-836, https://doi.org/10.1021/cn500101j (2014).

28. Liliang, P. C. et al. Relationship between injury severity and serum tau protein levels in traumatic brain injured rats. Resuscitation $\mathbf{8 1}$, 1205-1208, https://doi.org/10.1016/j.resuscitation.2010.05.016 (2010). 
29. Zetterberg, H. \& Blennow, K. From Cerebrospinal Fluid to Blood: The Third Wave of Fluid Biomarkers for Alzheimer's Disease. Journal of Alzheimer's disease: JAD 64, S271-s279, https://doi.org/10.3233/jad-179926 (2018).

30. Molinuevo, J. L. et al. Current state of Alzheimer's fluid biomarkers. Acta neuropathologica 136, 821-853, https://doi.org/10.1007/ s00401-018-1932-x (2018).

31. Zetterberg, H. Blood-based biomarkers for Alzheimer's disease-An update. Journal of neuroscience methods, https://doi. org/10.1016/j.jneumeth.2018.10.025 (2018).

32. Blennow, K. \& Zetterberg, H. Biomarkers for Alzheimer's disease: current status and prospects for the future. Journal of internal medicine 284, 643-663, https://doi.org/10.1111/joim.12816 (2018).

33. Baird, A. L., Westwood, S. \& Lovestone, S. Blood-Based Proteomic Biomarkers of Alzheimer's Disease Pathology. Frontiers in neurology 6, 236, https://doi.org/10.3389/fneur.2015.00236 (2015).

34. Chiu, M. J. et al. Multi-Channel SQUID-Based Ultra-High-Sensitivity In-Vitro Detections for Bio-Markers of Alzheimer's Disease Via Immunomagnetic Reduction. IEEE Transactions on Applied Superconductivity 21, 477-480, https://doi.org/10.1109/ TASC.2010.2087304 (2011).

35. Verberk, I. M. W. et al. Plasma Amyloid as Prescreener for the Earliest Alzheimer Pathological Changes. 84, 648-658, https://doi. org/10.1002/ana.25334 (2018)

36. Nabers, A. et al. Amyloid blood biomarker detects Alzheimer's disease. 10, https://doi.org/10.15252/emmm.201708763 (2018).

37. Zetterberg, H. et al. Plasma tau levels in Alzheimer's disease. Alzheimer's research \& therapy 5, 9, https://doi.org/10.1186/alzrt163 (2013).

38. Mielke, M. M. et al. Plasma phospho-tau181 increases with Alzheimer's disease clinical severity and is associated with tau- and amyloid-positron emission tomography. Alzheimer's \& dementia: the journal of the Alzheimer's Association 14, 989-997, https://doi. org/10.1016/j.jalz.2018.02.013 (2018).

39. Lambert, J. C. et al. Association of plasma amyloid beta with risk of dementia: the prospective Three-City Study. Neurology 73, 847-853, https://doi.org/10.1212/WNL.0b013e3181b78448 (2009).

40. Song, F. et al. Meta-analysis of plasma amyloid- $\beta$ levels in Alzheimer's disease. Journal of Alzheimer's disease: JAD 26, 365-375, https://doi.org/10.3233/jad-2011-101977 (2011).

41. Sobow, T., Flirski, M., Kloszewska, I. \& Liberski, P. P. Plasma levels of alpha beta peptides are altered in amnestic mild cognitive impairment but not in sporadic Alzheimer's disease. Acta neurobiologiae experimentalis 65, 117-124 (2005).

42. Assini, A. et al. Plasma levels of amyloid beta-protein 42 are increased in women with mild cognitive impairment. Neurology 63, 828-831 (2004)

43. Hanon, O. et al. Plasma amyloid levels within the Alzheimer's process and correlations with central biomarkers. Alzheimer's \& dementia: the journal of the Alzheimer's Association 14, 858-868, https://doi.org/10.1016/j.jalz.2018.01.004 (2018).

44. Scheuner, D. et al. Secreted amyloid beta-protein similar to that in the senile plaques of Alzheimer's disease is increased in vivo by the presenilin 1 and 2 and APP mutations linked to familial Alzheimer's disease. Nature medicine 2, 864-870 (1996).

45. Kosaka, T. et al. The beta APP717 Alzheimer mutation increases the percentage of plasma amyloid-beta protein ending at A beta42(43). Neurology 48, 741-745 (1997).

46. Schupf, N. et al. Elevated plasma beta-amyloid peptide Abeta(42) levels, incident dementia, and mortality in Down syndrome. Archives of neurology 64, 1007-1013, https://doi.org/10.1001/archneur.64.7.1007 (2007).

47. Mehta, P. D. et al. Increased plasma amyloid beta protein 1-42 levels in Down syndrome. Neuroscience letters 241, 13-16 (1998).

48. Ertekin-Taner, N. et al. Linkage of plasma Abeta42 to a quantitative locus on chromosome 10 in late-onset Alzheimer's disease pedigrees. Science (New York, N.Y.) 290, 2303-2304, https://doi.org/10.1126/science.290.5500.2303 (2000).

49. Hansson, O. et al. Evaluation of plasma Abeta as predictor of Alzheimer's disease in older individuals without dementia: a population-based study. Journal of Alzheimer's disease: JAD 28, 231-238, https://doi.org/10.3233/jad-2011-111418 (2012).

50. Ertekin-Taner, N. et al. Plasma amyloid beta protein is elevated in late-onset Alzheimer disease families. Neurology 70, 596-606, https://doi.org/10.1212/01.wnl.0000278386.00035.21 (2008).

51. Chouraki, V. et al. Plasma amyloid-beta and risk of Alzheimer's disease in the Framingham Heart Study. Alzheimer's \& dementia: the journal of the Alzheimer's Association 11, 249-257.e241, https://doi.org/10.1016/j.jalz.2014.07.001 (2015).

52. Graff-Radford, N. R. et al. Association of low plasma Abeta42/Abeta40 ratios with increased imminent risk for mild cognitive impairment and Alzheimer disease. Archives of neurology 64, 354-362, https://doi.org/10.1001/archneur.64.3.354 (2007).

53. Seppala, T. T. et al. Plasma Abeta 42 and Abeta 40 as markers of cognitive change in follow-up: a prospective, longitudinal, population-based cohort study. Journal of neurology, neurosurgery, and psychiatry 81, 1123-1127, https://doi.org/10.1136/ jnnp.2010.205757 (2010).

54. Fei, M., Jianghua, W., Rujuan, M., Wei, Z. \& Qian, W. The relationship of plasma Abeta levels to dementia in aging individuals with mild cognitive impairment. Journal of the neurological sciences 305, 92-96, https://doi.org/10.1016/j.jns.2011.03.005 (2011).

55. Koyama, A. et al. Plasma amyloid-beta as a predictor of dementia and cognitive decline: a systematic review and meta-analysis. Archives of neurology 69, 824-831, https://doi.org/10.1001/archneurol.2011.1841 (2012).

56. Toledo, J. B. et al. Factors affecting A $\beta$ plasma levels and their utility as biomarkers in ADNI. Acta neuropathologica 122, 401-413, https://doi.org/10.1007/s00401-011-0861-8 (2011).

57. Mattsson, N. et al. Plasma tau in Alzheimer disease. Neurology 87, 1827-1835, https://doi.org/10.1212/wnl.0000000000003246 (2016).

58. Dage, J. L. et al. Levels of tau protein in plasma are associated with neurodegeneration and cognitive function in a population-based elderly cohort. Alzheimer's \& dementia: the journal of the Alzheimer's Association 12, 1226-1234, https://doi.org/10.1016/j. jalz.2016.06.001 (2016).

59. Wang, T. et al. The efficacy of plasma biomarkers in early diagnosis of Alzheimer's disease. International journal of geriatric psychiatry 29, 713-719, https://doi.org/10.1002/gps.4053 (2014).

60. Sparks, D. L. et al. Tau is reduced in AD plasma and validation of employed ELISA methods. American journal of neurodegenerative disease 1, 99-106 (2012).

61. Cosentino, S. A. et al. Plasma ss-amyloid and cognitive decline. Archives of neurology 67, 1485-1490, https://doi.org/10.1001/ archneurol.2010.189 (2010)

62. Llado-Saz, S., Atienza, M. \& Cantero, J. L. Increased levels of plasma amyloid-beta are related to cortical thinning and cognitive decline in cognitively normal elderly subjects. Neurobiology of aging 36, 2791-2797, https://doi.org/10.1016/j. neurobiolaging.2015.06.023 (2015).

63. Pomara, N., Willoughby, L. M., Sidtis, J. J. \& Mehta, P. D. Selective reductions in plasma Abeta 1-42 in healthy elderly subjects during longitudinal follow-up: a preliminary report. The American journal of geriatric psychiatry: official journal of the American Association for Geriatric Psychiatry 13, 914-917, https://doi.org/10.1176/appi.ajgp.13.10.914 (2005).

64. Blasko, I. et al. Conversion from cognitive health to mild cognitive impairment and Alzheimer's disease: prediction by plasma amyloid beta 42, medial temporal lobe atrophy and homocysteine. Neurobiology of aging 29, 1-11, https://doi.org/10.1016/j. neurobiolaging.2006.09.002 (2008). 


\section{Acknowledgements}

This work was supported by Taiwan Ministry of Science and Technology grants (MOST 107-2314-B-010-002 and MOST107-2320-B-010-043), the National Health Research Institutes (NHRI-EX106-10614NI), a Taiwan Ministry of Education Aim for the Top University Grant, Taipei Veterans General Hospital (V105C-074 and V107C-090), and the Brain Research Center at the National Yang-Ming University from The Featured Areas Research Center Program within the framework of the Higher Education Sprout Project by the Ministry of Education (MOE) in Taiwan.

\section{Author Contributions}

T.B.C., C.J.H., P.N.W. and I.H.C. contributed to the conception and design of the study, acquisition of data, interpretation of the data, and critical revision of the manuscript. Y.J.L. and S.Y.L. were involved in the collection and/or analysis of data. J.P.C. performed statistical analysis. T.B.C. and P.N.W. wrote the first draft of the manuscript. All authors read and approved the submitted version.

\section{Additional Information}

Competing Interests: The authors declare no competing interests.

Publisher's note Springer Nature remains neutral with regard to jurisdictional claims in published maps and institutional affiliations.

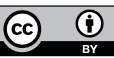

Open Access This article is licensed under a Creative Commons Attribution 4.0 International License, which permits use, sharing, adaptation, distribution and reproduction in any medium or format, as long as you give appropriate credit to the original author(s) and the source, provide a link to the Creative Commons license, and indicate if changes were made. The images or other third party material in this article are included in the article's Creative Commons license, unless indicated otherwise in a credit line to the material. If material is not included in the article's Creative Commons license and your intended use is not permitted by statutory regulation or exceeds the permitted use, you will need to obtain permission directly from the copyright holder. To view a copy of this license, visit http://creativecommons.org/licenses/by/4.0/.

(C) The Author(s) 2019 